\title{
Les aveux et dénombrements du Vendômois : réalités et représentations (1311-1550)
}

\section{Christophe Marion}

\section{(2) OpenEdition}

1 Journals

\section{Édition électronique}

URL : http://journals.openedition.org/abpo/1370

DOI : $10.4000 /$ abpo. 1370

ISBN : 978-2-7535-1491-1

ISSN : 2108-6443

Éditeur

Presses universitaires de Rennes

Édition imprimée

Date de publication : 20 octobre 2003

Pagination : $55-76$

ISBN : 978-2-86847-929-7

ISSN : 0399-0826

\section{Référence électronique}

Christophe Marion, «Les aveux et dénombrements du Vendômois : réalités et représentations

(1311-1550) », Annales de Bretagne et des Pays de l'Ouest [En ligne], 110-3 | 2003, mis en ligne le 20 octobre 2005, consulté le 19 avril 2019. URL : http://journals.openedition.org/abpo/1370 ; DOI : 10.4000/abpo. 1370 


\title{
Les aveux et dénombrements du Vendômois : réalités et représentations (1311-1550)
}

\author{
Christophe MARION \\ PRAG à l'université de Tours \\ Doctorant au Laboratoire de médiévistique occidentale de Paris, université Paris 1
}

\section{" Les mots ne sont pas d'emblée traduction des idées "}

Les aveux et dénombrements forment un corpus connu des médiévistes. Les érudits du XIX ${ }^{\mathrm{e}}$ siècle s'en inspirèrent afin de rédiger les dictionnaires locaux dont nous nous servons encore si souvent (il s'agissait la plupart du temps de localiser le fief et de dresser la liste, plus ou moins complète, des feudataires ${ }^{1}$ ). Les historiens de la seigneurie ou de la société firent des conquêtes bourgeoises, des permanences nobiliaires, de l'effondrement ou de la consolidation du patrimoine lignager leur domaine d'études. Enfin, et plus récemment, les archéologues notèrent avec minutie les mentions concernant l'habitat ou le parcellaire, complétant ainsi les études de terrain et des cadastres. Si les utilisateurs ont été divers et nombreux, tous ont déploré la stéréotypie de l'acte, condamné puisque soumis au " critère subjectif de l'originalité ${ }^{2}$ ", oubliant ainsi que l'innovation médiévale pouvait prendre des formes bien anodines ${ }^{3}$. Les uns constataient que la reprise

1. Les dictionnaires de Raoul de Saint-Venant pour le Vendômois (SAINT-VENANT, Raoul Barré de, Dictionnaire topographique, historique, biographique, généalogique et héraldique du Vendômois et de l'arrondissement de Vendôme, 4 vol., Blois, 1912-1917), de Carré de Busseroles pour la Touraine (Busserolles, Jacques-Xavier Carré de, Dictionnaire géographique, historique et biographique d'Indre-et-Loire et de l'ancienne province de Touraine, $6 \mathrm{vol}$., Tours, 1878-1884), de Célestin Port pour le Maine-et-Loire (PORT, Célestine, Dictionnaire historique, généalogique et bibliographique de la province et du diocèse du Mans, Mayenne, 1895) ou de Eugène Vallée (VALLEE, Eugène, Dictionnaire topographique, historique et statistique de la Sarthe, 6 vol., Le Mans, 1829-1842) sont des outils dont le maniement est encore indispensable pour qui veut appréhender le comté de Vendôme à la fin du Moyen Âge.

2. ZimMERMANN, Michel (dir.), Auctor et auctoritas. Invention et conformisme dans l'écriture médiévale, Mémoires et documents de l'École des Chartes, n 59 , Paris, École des Chartes, 2001, p. 12.

3. "L'innovation ne réside pas dans la création de nouveaux thèmes, mais bien dans la réorganisation permanente de motifs anciens " : BELMON, Jérôme, «L'écriture des actes 
des aveux antérieurs rendait difficile l'utilisation d'un document qui traduisait des réalités d'une autre époque. Les autres étaient très vite déçus par les résultats qu'offraient la comparaison entre données de terrain d'une part et description, très vague, du dénombrement d'autre part : les mêmes questions revenaient ainsi sans cesse, notamment celles concernant la réalité matérielle de l'habergement, de la métairie ou du chastel ${ }^{4}$.

Toutefois, malgré ces critiques, chacun a continué d'utiliser l'aveu, réputé objectif ${ }^{5}$, qui apportait de nombreuses indications sur la terre (plusieurs études se sont attachées à décrire les ravages de la guerre de Cent Ans à travers les mentions de tenures ou de fiefs en gast) et les hommes. Ces derniers, qualifiés d'écuyer ou de chevalier, intégraient le groupe des nobles alors que l'officier, le bourgeois et le marchand rejoignaient celui des "roturiers conquérants". Lorsqu'il avait la chance de suivre sur plusieurs décennies les destinées du feudataire, l'historien se trouvait confronté à des titulatures hésitantes qui induisaient ou semblaient induire un changement important du statut social. Il y aurait mauvaise grâce à nier la belle ascension sociale de la famille Lespine alors que Pierre, non titré en 1397, était écuyer en 1409, avant que son fils n'accède à la chevalerie ${ }^{6}$. Toutefois, la question du crédit que l'on peut accorder à l'acte doit se poser. Que Pierre de Lespine se présente comme écuyer en 1397 est un fait documentaire : s'agit-il pour autant d'en faire un noble? Nous n'avons pas toujours été aussi prudents que les sociologues qui, tels Pierre Bourdieu, ont insisté sur le danger qu'il y a à ratifier purement et simplement " les représentations que les agents sociaux se font de la réalité " (même s'il est évident que "leur vision et leurs interprétations sont une composante incontournables de la réalité complète du monde social ") ${ }^{7}$. Ces remarques demeureraient valables dès que l'on s'intéresse au fief. Celui de Vaulevrier, en la paroisse de Villiers, est assez bien connu, grâce à une série de neuf aveux et dénombrements, répartis entre 1374 et 1516. A la fin du XIV siècle, le vassal avouait ce qu'il tenait " en fie et en domaine ". Ses successeurs décrivirent leur " habergement ", puis leur " terre et seigneurie " et enfin

de la pratique en Languedoc et en Toulousain (IX ${ }^{\mathrm{e}}$-XI ${ }^{\mathrm{e}}$ siècle) ", ZimmERMANN, Michel, Auctor et auctoritas..., op. cit., p. 308; " Prévaut généralement chez les historiens l'idée que les actes de la pratique, ce que l'on nomme, après les historiens allemands, l'écriture pragmatique, sont caractérisés par une stéréotypie et un conformisme qu'on explique par la soumission à des formulaires ", Zimmermann, Michel, "Vie et mort d'un formulaire. L'écriture des actes catalans (X $\mathrm{X}^{\mathrm{e}}$-XII ${ }^{\mathrm{e}}$ siècle) ", Zimmermann, Michel, Auctor et auctoritas..., op. cit., p. 337.

4. Les historiens, avant les archéologues, ont souligné l'ambiguïté des termes employés dans les dénombrements. Marie-Thérèse Caron notait qu'il était difficile de séparer meix et domaine, domaine et seigneurie... CARON, Marie-Thérèse, La Noblesse dans le duché de Bourgogne (1315-1477), Lille, 1987, p. 463.

5. Sur l'objectivité des actes de la pratique, voir ZimMERMAnN, Michel, Auctor et auctoritas..., op. cit., p. 13.

6. Arch. nat., P 627 n $^{\circ} 52$ (1397), nº 53 (1409), nº 54 (1436).

7. BouRdieu, Pierre, Ce que parler veut dire. L'économie des échanges linguistiques, Paris, Fayard, 1982, p. 147. 
leur " fief et seigneuri ${ }^{8}$ ». Or, face à ces changements lexicaux repérables à une plus vaste échelle, les dénombrements ne traduisent aucun changement notable dans le contenu du bien déclaré. Pendant longtemps, ces diverses procédures d'identification ont été soit ignorées ${ }^{9}$, soit considérées comme des révélateurs d'une réalité préexistante. Elles furent rarement envisagées comme contribuant à construire cette même réalité. La question de savoir si l'apparition de la seigneurie reflète un changement social revient à s'interroger sur la portée des transformations lexicales : démontrent-elles une réalité ou sont-elles la projection documentaire d'une société désirée, rêvée, par les contemporains (une image en quelque sorte, non pas reflet mais instrument du changement)?

\section{Le corpus des aveux et dénombrements}

Nous avons consulté dix registres conservés aux Archives nationales et contenant les originaux des aveux et dénombrements rendus au comte entre 1350 et $1550^{10}$. Le classement semble dater du XVIII ${ }^{\mathrm{e}}$ siècle, alors que les documents de la chambre des comptes de Vendôme furent transférés à Paris : les dénombrements d'un même fief sont rassemblés, et classés du plus récent au plus ancien, même si les exceptions sont nombreuses. Plusieurs types d'actes peuvent être différenciés :

- deux " enquêtes de fiefs ", rédigées en 1311 et $1355^{11}$, semblables à l'enquête ordonnée en Bourgogne par Charles le Téméraire (1474) et étudiée par M.-Th. Caron ${ }^{12}$;

- les aveux suivis ou non d'un dénombrement, et parmi ceux-ci : les " aveux directs ", c'est-à-dire les actes dans lesquels le vassal, à la première personne du singulier, s'avouait l'homme de son seigneur; les " aveux indirects " enfin, dans lesquels le vassal n'était pas présent lors du serment : devant une cour voisine (très souvent celle de Châteaudun ou celle de Blois), il avait dénombré sa terre. Ces actes, minoritaires, étaient rédigés à la troisième personne du singulier ${ }^{13}$.

La rédaction des aveux fut probablement liée à la situation politique difficile que traversa le royaume durant le bas Moyen Âge ${ }^{14}$. La brusque augmentation du nombre d'actes durant les décennies 1390-1410 pourrait être

8. Arch. nat., $\mathrm{P} 601 \mathrm{n}^{\circ} 10$ (1374), $\mathrm{n}^{\circ} 11$ (1401), n 12 (1414), $\mathrm{n}^{\circ} 13$ (1438), $\mathrm{n}^{\circ} 14$ (1455), $n^{\circ} 15$ (1484), $n^{\circ} 16$ (1488), $n^{\circ} 18(1516), n^{\circ} 103$ (1511).

9. R. de Saint-Venant n'a jamais hésité à traduire " écuyer " ou " chevalier " par noblesse, " métairie " ou " habergement " par fief et seigneurie.

10. Arch. nat., P 601, P 611, P 618, P 621, P 623, P 625, P 627, P 639, P 652, P 657.

11. Arch. nat., P 976, 1355 (vassaux de la châtellenie de Vendôme); P 973, xIve siècle (vassaux du comté).

12. CARON, Marie-Thérèse, La Noblesse..., op. cit.

13. "Saichent tous présents, Nous justice de la chastellenie de Marchesnoir, présente noble dame Jehanne Belon veuve de messire Alart de Courbenton jadis chevalier confesse qu'elle tient, tenir en fief de haut et puissant seigneur monsieur le comte de Vendosme... ». Arch. nat., P 639, n 56, 1405.

14. Cf. Annexe. 
comparée avec la même inflation de baux rédigés par les chanoines de la collégiale Saint Georges durant cette période ${ }^{15}$. Elle doit être interprétée comme un signe, parmi d'autres, de la remise en ordre qui suivit la première vague d'opérations militaires de la guerre de Cent Ans. Convalescence des campagnes, remise en ordre administrative ressortiraient d'une même volonté de reconstruction. Des conclusions qui demeureraient valables pour les décennies 1430-1450 : les dénombrements se firent alors censiers et même terriers. La liste des censitaires, de leurs tenures et de leurs obligations (en cens et en rentes) fut dressée par le vassal. Il n'est pas rare de voir plus de la moitié de l'acte réservée à cette description, très souvent minutieuse (les confronts des parcelles par exemple étaient détaillés) ${ }^{16}$. Deux dates doivent être placées à part : 1484 et 1516 correspondent en effet à deux années riches en étiquettes ${ }^{17}$. Dans le premier cas, le comté de Vendôme venait d'être soustrait à la vassalité angevine et placé directement sous la seigneurie royale ${ }^{18}$. Dans le second cas, le comté était érigé en duché par François Ir $^{19}$. À cette occasion, les vassaux de François de Bourbon et Charles ${ }^{\text {er }}$, probablement sur leur ordre, déclarèrent leur fief. C'est le seul moment où nous constatons un lien aussi étroit entre l'autorité publique et la rédaction d'aveux : si le cheval de service était payable à mutation d'homme ou de seigneur, force est de constater que le décès d'un comte ne s'accompagna jamais d'une prestation d'aveux ${ }^{20}$. La rédaction de ces derniers a donc été vraisemblablement commandée par des motifs juridiques (le changement de statut du comté) et économiques (une volonté de remise en ordre après la guerre).

L'hommage, nous y reviendrons, était un serment oral : l'aveu reprenait le serment du vassal et le dénombrement détaillait par le menu le contenu du fief. Les premiers aveux et dénombrements conservés ne sont pas antérieurs à 1350. Toutefois, la situation à l'aube du XIve siècle peut être appré-

15. MARION, Christophe, La Reconstruction des campagnes vendômoises à travers l'exemple des domaines de la collégiale Saint-Georges de Vendôme, Mémoire de Maîtrise, Université de Tours, 1995.

16. En 1458, Jehan de Vaulevrier déclarait son habergement de Vaulevrier : environ 1500 mots (sur 2500 mots que comptait l'aveu) étaient consacrés à la liste des censitaires et des tenanciers qui devaient payer le terrage (la terre était décrite avec précision), Arch. nat., P 601, n 14, 1455.

17. Sous ce terme, il faut entendre des aveux, rédigés sur papier, et non accompagnés de dénombrements.

18. La baronnie de Mondoubleau était dans la mouvance du comté du Maine; le comté de Vendôme dans celle du duché d'Anjou. "Par considération de la proximité du lignage et aussi des grans louables proffitables et recommandables services que ses prédécesseurs comtes de Vendosme ont par longtemps faits à nos prédécesseurs ", Charles VIII assemblait comté de Vendôme et baronnie de Mondoubleau qui devenaient comté de Vendosmois. De plus, pour ce nouvel ensemble, un seul hommage était rendu directement à la couronne de France. Bibliothèque nationale : ms fr 16648, 1484.

19. Bibliothèque nationale : ms fr 2758.

20. L'accession des Bourbon à la tête du comté (1371), la mort de Jean VII (1393), de Louis $\mathrm{I}^{\mathrm{er}}$ (1446), de Jean VIII (1477), de François (1495) ou de Charles I $^{\mathrm{er}}$ (1537) ne furent pas accompagnés d'un accroissement notable du nombre d'aveux et dénombrements. 
hendée grâce à une liste d'hommages des vassaux vendômois compilée par la comtesse douairière quelques décennies plus tard (" Ce sont les homages monsieur le comte reçus l'an 1311 si comme Hameau les apporta à la dicte dame ") ${ }^{21}$. Cette énumération, probablement rédigée comme le Livre des fiefs de 1355 à l'aide des actes conservés par la chancellerie du château, met en lumière une époque où l'administration du comte peinait à sortir de l'oralité. Si Jehan de Chaufourneau était " home de foy " pour " un estre au Touchet (Lunay) et un quartier et demi de vigne à la Huraudière et trois minées de terre en la varenne d'Aunays ", Bouchard de Villeprouvaire se contentait de déclarer " ce qu'il tient à Villiersfaux ". Guillaume de Rougemont tenait « une métairie à Périgny à un cheval de service ", mais Georges Poinvillain devait simplement "six fois [sic] et hommages tant pour Montoire, Lavardin comme Trôo " (sous entendu pour ce qu'il tenait dans ces paroisses du Bas-Vendômois). Ces hésitations entre l'hommage, serment oral qui n'inventoriait probablement pas le fief, et l'aveu écrit accompagné d'un dénombrement précis, rappellent la dichotomie entre écriture et oralité mise en lumière par J. Goody ${ }^{22}$. Si l'énumération de 1311 hésitait entre hommage et aveu, oralité et écriture, il semble que le Livre des fiefs résolut le problème au profit du dernier choix : en 1355 en effet, le registre de Jacquet du Pont recopiait des dénombrements précis, non de simples hommages. Toutefois la victoire de ce type d'acte, si elle illustre pour une part l'utilisation plus fréquente de l'écriture dans les rapports féodo-vassaliques, fut assez longue et jamais totale. Durant la seconde moitié du XIV ${ }^{\mathrm{e}}$ siècle, six vassaux déclaraient sans détail "les choses dont je suis en vostre foy et homage tant fie comme domaines ", rappelant ainsi certaines formulations vagues de $1311^{23}$. De même, les années 1484 et 1516 furent deux moments importants durant lesquels furent rédigés plusieurs dizaines d'aveux sans dénombrements dont la présence pose plusieurs problèmes qu'il nous faudra tenter d'élucider.

L'aveu et dénombrement conservé aux Archives nationales était l'aboutissement d'une succession de rites, dont le détail nous échappe. L'hommage était oral : le serment, prêté lors des assises du comté, nous est connu par les registres judiciaires du Vendômois (dont aucun n'est antérieur à 1450) ${ }^{24}$. En 1455 "Françoise de Crassay veuve de Michelet Vendômois a juré la féaulte des foy et homage qu'elle doit pour raison des domaines du Cloux Margot, de Touchard et des appartenances. Elle a

21. Arch. nat., P 973 (dénommé Livre d'Alix de Bretagne par Dominique Barthélemy), XIV siècle.

22. Jack Goody a beaucoup travaillé sur le concept de liste. Pour lui la liste différait beaucoup de la communication orale. Elle n'était pas une représentation directe de la parole, qui s'accommodait mal d'une énumération longue et fastidieuse ('inventaire d'un domaine par exemple, dans le cas d'un aveu et dénombrement) : il en soulignait donc l'absence dans les sociétés orales qui ne maîtrisaient pas l'écriture. Goody, Jack, La raison graphique. La domestication de la pensée sauvage, Paris, Minuit, 1979, p. 140 sqq.

23. Ces vassaux appartenaient à d'anciennes familles de l'aristocratie (ils portaient d'ailleurs tous l'armigérat) : les Saint-Amand, Marray, Vendôme, Belon, Noce et Marquier.

24. Arch. nat., P 984, remembrances d'assises de Mondoubleau (1451-1457). 
reconnu un cheval de service et nous l'avons enjoint à bailler adveu par temps de coustume ". Cette première étape était, à notre sens, fondamentale. D'une part, parce que la prestation de serment était publique : les assises rassemblaient beaucoup de monde ${ }^{25}$ et la qualité de vassal apparaissait alors d'une manière évidente. D'autre part, parce qu'elle sanctionnait, légitimait, une différence sociale. En cela, nous ferions de l'hommage un véritable " rite d'institution ", qui transformait réellement la personne consacrée mais également la représentation que s'en faisait les autres ${ }^{26}$. Certes, l'institution (c'est-à-dire ici les assises) ne créait pas une différence ex nihilo mais exploitait celles qui existaient déjà, comme des écarts de richesse, de revenus ou de capital symbolique. Mais le fait est là : les assises comtales instituaient le vassal, dont la qualité était d'autant moins contestée que l'institution était reconnue de tous (en cela, l'étude de l'hommage ne saurait être séparée des progrès de l'autorité publique ${ }^{27}$ ). Quelques semaines après le serment, toujours lors des réunions judiciaires de la châtellenie, le dénombrement était solennellement remis au bailli seigneurial, présenté " davantage aux yeux qu'aux oreilles " (nous ne pensons pas que l'inventaire fastidieux du fief était lu lors de la cérémonie). Ainsi, le rite était conclu par la remise d'un document dont la matérialité, sans aucun doute porteuse de sens, mériterait une étude soignée ${ }^{28}$. Tous les aveux utilisaient le parchemin, véhicule idéal de la perpétuité et de l'authenticité que les auteurs souhaitaient attacher au rite. Certains étaient scellés, d'autres non ${ }^{29}$. Enfin, le prestige du vassal était-il identique lorsqu'il remettait un parchemin long de 20 centimètres ou de plus de 4 mètres ${ }^{30}$ (l'effet visuel

25. Roberde de Saint Berthenin, dame de la Chesnaye, avait quitté un peu précipitamment les assises de Mondoubleau en 1453 pour manifester son rejet du jugement qui lui était peu favorable. Obligée de revenir à de meilleurs sentiments, elle avait été sommée d'expliquer son départ : " en disoit qu'elle ne cudoit pas offencer de l'autorité... qu'elle s'en estoit ainsi hier au soir allée sans prendre congié de nous pour doubte qu'elle trouvast pas logeis compétent tant parce qu'il y avoit grant nombre de gens pour cause des assises ». Arch. nat., P 984, remembrances d'assises de Mondoubleau (1451-1457).

26. Bourdieu, Pierre, Ce que parler veut dire..., op. cit., p. 121-134.

27. Pierre Bourdieu affirme que pour comprendre le pouvoir des expressions linguistiques, il ne faut pas s'intéresser uniquement au langage mais également et surtout "à l'autorité qui donne force à ce langage ": ici, l'autorité seigneuriale. BouRDIEU, Pierre, Ce que parler veut dire... op. cit., p. 121-134.

28. Les historiens de la poésie japonaise ont souligné combien le waka (un poème oral à l'origine) était entré, par le biais de l'écriture, dans le monde de la plastique : Jacqueline Pigeot notait qu'il fournissait un aspect seulement du message, illisible dans son intégralité si le lecteur ne s'intéressait pas au paratexte constitué par le support, le messager (PIGEOT, Jacqueline, " La poésie japonaise entre écriture et vocalité ", ALLETON, Viviane, Paroles à dire, Paroles à écrire. Inde, Chine, Japon, Paris, Éditions de l'EHESS, 1997, p. 42).

29. L'étude sigillographique paraît essentielle : certains vassaux faisaient authentifier l'acte par le "scel aux contrats de la chastellenie de Vendosme ». D'autres utilisaient leur propre sceau (" mon scel de mes armes").

30. Dénombrement de la terre de Villeromain, rendu en 1469 par Hardouin de Maillé, chevalier et seigneur de Villeromain. Arch. nat., P 618, ${ }^{\circ}$ 57, 1469. Les aveux les plus longs étaient généralement associés à des seigneuries anciennes, à des lignages pluriséculaires. 
devait être probablement différent même si le classement des documents issus de la chambre des Comptes de Vendôme dans des registres au XVIII ${ }^{\mathrm{e}}$ siècle nous empêche de connaître le pliage originel)?

\section{Inscrire l'individu dans un lignage}

Le dénombrement était conservé ensuite par les archivistes du comte mais également par le vassal. Il servait à rédiger les dénombrements suivants $^{31}$ mais également à justifier un droit sur la terre. Entre 1377 et 1459, les vassaux qui avouèrent les moulins de la Chappe et la justice de Chanteloup utilisèrent deux " formulaires ". L'un mentionnait " deux moulins à blé, appelés les moulins de la Chappe; ma justice en ma terre, domaine et fiefs de Chanteloup "; diverses clauses finales énuméraient les garanties, les précautions prises pour l'application de l'acte : "Pleige gage droit obéissance; protestation de bailler de bouche, par écrit, montre ou autrement plus à plain. " L'autre différait quelque peu en indiquant " un moulin à blé sur la rivière du Loir et la place d'un autre moulin auprès d'icelui en la basse Chappe; des foy et hommages; la haute justice en ma terre de Chanteloup ". Les clauses finales mentionnaient la formule "pleige, droit, gage, sujétion, obéissance ». En 1377, Jehan de Beauvoir utilisa le premier type ${ }^{32}$. Son successeur Bertrand Daguier, époux de sa petite fille Agnès, reprit le même en 1397 mais utilisa la seconde version en $1409^{33}$. Le nouvel époux d'Agnès, Louppes de Tallentes, utilisa la formulation de Daguier en 1433 alors que son aveu lui avait probablement été transmis ${ }^{34}$. Toutefois Jehan Daguier, fils de Bertrand et d'Agnès, copia le dénombrement de Beauvoir en $1459^{35}$. Ainsi, il se rattachait à la grande famille des Malon, à un double titre : il se proclamait " fils aisné de feu Bertrand Daguier et de Agnès la Maslonne " et utilisait une formulation ancienne, dont l'origine devait être placée à la fin du XIV ${ }^{e}$ siècle, lorsque la terre était possédée par Jean de Beauvoir "à cause de sa femme " (veuve de Gervais Malon) et, avant lui, Gervais Malon. Cet exemple nous montre que le dénombrement circulait, changeait de mains à l'occasion des partages mais de manière raisonnée, calculée : véritable titre de propriété et pourvoyeur de "capital symbolique ", il permettait d'affirmer un rattachement à d'anciens lignages. Après le décès de Bertrand, sa veuve avait probablement reçu en partage un dénombrement moins prestigieux, comme pour mieux lui manifester que ses droits étaient limités. Le douaire pesait bien peu face aux prétentions du fils aîné, seul véritable héritier et unique détenteur de l'acte sécu-

31. En 1440, Jean Georget avouait sa métairie de Champgast (Crucheray) à Aipzon d'Estouteville et précisait " item j'ai trouvé par aucuns escrits anciens que doy autre dismes à Espinoches qui souloient valoir anciennement un muide de blé par chascun an "; Arch. nat., P 611, n 37, 1440.

32. Arch. nat., $\mathrm{P} 627, \mathrm{n}^{\circ} 32,1377$.

33. Arch. nat., P 627, $\mathrm{n}^{\circ} 34,1409 ; \mathrm{n}^{\circ} 33,1397$.

34. Arch. nat., P 627, $\mathrm{n}^{\circ} 36,1433$.

35. Arch. nat., $\mathrm{P} 627, \mathrm{n}^{\circ} 37,1459$. 
laire. Nous le voyons, la réutilisation des aveux antérieurs n'était pas anodine. Loin de manifester la perplexité du vassal, son incompétence ou sa peur de ne pas employer les bons termes à une époque où le sens juridique de chaque mot devait être soigneusement pesé, elle participe probablement de cette volonté de replacer l'individu dans un lignage, de rattacher le présent à un passé dont il serait, pour partie, héritier ${ }^{36}$. L'étude des mentions de parenté confirme cette impression.

À quarante-cinq reprises, le vassal mentionnait qu'il tenait sa terre « à cause de sa femme " (la plupart du temps, prénommée, voire nommée, sans autre détail). Toutefois, dans quelques cas ${ }^{37}$, l'aveu amorçait une véritable généalogie, en indiquant, à côté du patronyme de l'épouse, celui de son père : ces exemples concernent les terres de Maugué, Chanteloup et la Chappe (la Chapelle Anschéry, Vendôme, Villerable), Flamesec (Villetrun), la Béginière (Champigny), le Boile (Pezou), Challay (Saint-Quentin), Vaulevrier (Villiers). Dans plus de 90 \% des cas, le vassal appartenait à la notabilité urbaine ${ }^{38}$ : avec l'aveu, il rappelait son alliance passée avec de grandes familles bourgeoises, comme les Malon ${ }^{39}$, Beauvoir ${ }^{40}$, Bégin ${ }^{41}$ ou Lefèvre $^{42}$. S'il n'est pas de notre propos de débattre de l'anoblissement par le fief, force est de constater que, lorsqu'un véritable système de titres se mettra en place (seconde moitié du $\mathrm{XV}^{\mathrm{e}}$ siècle, $\mathrm{XVI}^{\mathrm{e}}$ siècle), ces " généalogies " tendront à disparaître, comme si leur utilité était désormais contestée. La mention de l'armigérat ou de la chevalerie, écrite dans l'aveu, sera alors meilleure garante de noblesse que la possession du fief pendant deux ou trois générations. Les mêmes remarques demeureraient valables pour la mention de l'ascendant, même s'il est nécessaire de noter une nuance ${ }^{43}$ : alors que l'allusion au lignage de l'épouse concerne surtout la fin du

36. « De fait, quelle que soit la nature, la reprise de modèles anciens donne souvent à une charte une valeur mémorielle supplémentaire : en créant un lien particulier entre deux ou plusieurs actes, elle inscrit clairement leurs auteurs juridiques dans une succession familiale, dans un réseau de clientèle, dans une continuité de fonction, ou les rattache à un passé prestigieux ": BELMON, Jérôme, "L'écriture des actes de la pratique en Languedoc et en Toulousain (IX ${ }^{\mathrm{e}} \mathrm{XI}{ }^{\mathrm{e}}$ siècle) ", ZIMmERmann, Michel, Auctor et auctoritas..., op. cit., p. 308.

37. Arch. nat., $\mathrm{P} 618, \mathrm{n}^{\circ} 9,1397 ; \mathrm{n}^{\circ} 8,1409 ; \mathrm{n}^{\circ} 8$ bis, $1415 ; \mathrm{n}^{\circ} 6,1433 . \mathrm{P} 627, \mathrm{n}^{\circ} 33,1397$; $\mathrm{n}^{\circ} 34,1409 ; \mathrm{n}^{\circ} 36,1433 ; \mathrm{n}^{\circ} 103,1511 . \mathrm{P} 623, \mathrm{n}^{\circ} 86,1434 ; \mathrm{n}^{\circ} 39,1436 ; \mathrm{n}^{\circ} 23,1487 . \mathrm{P} 625$, $\mathrm{n}^{\circ} 25,1460 . \mathrm{P} 639, \mathrm{n}^{\circ} 62,1502$.

38. Le seul contre-exemple concerne «François de Mazangé, écuyer seigneur de Souday et Challay, à cause de Catherine de Saint-Béthenin, ma femme fille de feu Jehan de SaintBéthenin, écuyer et seigneur des dictes seigneuries ". Arch. nat., P 639, n 62, 1502.

39. " Je Bertrand Daguier, à cause de Agnès ma femme fille de feu Jehan Maslon l'aisné ", P $627, n^{\circ} 34,1409$.

40. "Je Mahiet Fiquet, à cause de ma femme Jehanne, fille de Henri Rebours et de Michelle fille de feu Jehan de Beauvoir ", P 623, n 86, 1484.

41. "Je Jehan Briençon, à cause de Périne fille de feu Jehan Bégin ma femme ", P 623, $\mathrm{n}^{\circ} 39,1436$.

42. "Je Jehan Bouvier, demourant à Vendosme, à cause de Denise ma femme fille de feu Guillaume Lefèvre ", P $623, n^{\circ} 23,1487$.

43. Le vassal se disait "fils de " : 27 cas dont la majorité concerne là aussi des vassaux issus de la notabilité vendômoise. 
$\mathrm{XIV}^{\mathrm{e}}$ siècle et la première moitié du siècle suivant, le patronyme du père se retrouve plus tardivement. Le passage d'une vision strictement agnatique ${ }^{44}$ des choses à une conscience patrilinéaire pourrait s'expliquer par les progrès de l'écrit ${ }^{45}$.

La conscience lignagère fut souvent considérée comme l'apanage de la noblesse ${ }^{46}$ : ici, elle concerne plus largement le groupe des "vassaux du comte " dont la division entre nobles et roturiers ne nous semble pas forcément toujours pertinente ${ }^{47}$.

\section{"Service vassalique " et " état de noblesse " : quelles réalités?}

L'historien médiéviste doit souvent étudier des hommes dont il ne sait quasiment rien. La tentation est grande d'additionner, pour un même individu, les différentes mentions qui le concernent, les titres qu'il porte à diverses occasions sans prendre soin de s'intéresser à la typologie des actes. Ainsi, une véritable destinée peut être écrite $a$ posteriori sans lien véritable avec la réalité sociale. La question se pose de la validité de cette méthode, qui consiste à accumuler les éclairages afin de dresser un portrait aussi proche que possible d'une certaine réalité médiévale. L'exemple des Beauxoncles pourra illustrer ces dangers. Si l'origine de la famille est inconnue, nous pensons pouvoir la rattacher à la notabilité urbaine ${ }^{48}$. Jean demeurait à Vendôme en 1392, était bourgeois en $1414^{49}$ et «noble homme

44. La vision selon laquelle le Moyen Âge aurait privilégié la patrilinéarité est contestée par de nombreux historiens : GuERREAU-JALABERT, Anita, article "Parenté ", LE Goff, Jacques, Schmitt, Jean-Claude (dir.), Dictionnaire raisonné de l'Occident médiéval, Paris, Fayard, 1999.

45. "Cette conscience de la patrilignée qui est allée en s'accentuant du XV ${ }^{\mathrm{e}}$ au XVII ${ }^{\mathrm{e}}$ siècle, était aussi favorisée par l'essor de l'écrit " : NASSIET, Michel, Parenté, noblesse et Etats dynastiques. XVe-XVII siècles, Paris, EHESS, 2000, p. 41.

46. Les généalogies princières du XII ${ }^{\mathrm{e}}$ siècle, comme celle de Baudouin V comte de Hainaut écrite par son chapelain, s'attachaient à exalter un lignage dont l'individu devait se montrer digne.

47. Le terme de " groupe social " semble adéquat à partir du moment où « la conscience d'une appartenance commune " nous paraît naître lors de la cérémonie de l'hommage. Pour la définition du terme : ÉTIENNE, Jean (dir.), Dictionnaire de sociologie. Les notions, les mécanismes et les auteurs, Initial, Hatier, Paris 1997, p. 180-184.

48. Les premières apparitions de Jehan Beauxoncles se situent en ville : en 1339, il échangeait une rente contre 5 quartiers de vigne dans la zone périurbaine (Arch. dép. de Loir-et-Cher, G 238, 1339). Il avait épousé Thenote Lefèvre (Bibliothèque municipale : ms 285), dont la famille se retrouve à plusieurs reprises au service du comte. R. de SaintVenant plongeait les racines des Beauxoncles dans le monde des tanneries : "Il y avait à Pezou aux XIV e et XV ${ }^{\mathrm{e}}$ siècles des tanneries. Ces tanneries au XIV ${ }^{\mathrm{e}}$ siècle étaient possédées par Jean Beauxoncles, bourgeois de Vendôme, dont la famille s'enrichit et fut anoblie ": SAINT-VEnAnt, Raoul Barré de, Dictionnaire topographique, historique, biographique, généalogique et héraldique du Vendômois et de l'arrondissement de Vendôme, 4 vol., Blois, 19121917, III, p. 45.

49. Arch. dép. de Loir-et-Cher G 267, 1414 : « Jehan Beauxoncles, bourgeois de Vandosme confesse qu'il a reçu des chanoines de la collégiale Saint-Georges par la main de Jehan Rouegmont, la somme de 60 livres tournois". 
monsieur en $1393^{50}$ ». Son fils Jacques, chevalier en $1425^{51}$, avait épousé une femme issue d'un très ancien lignage chevaleresque (les Saint-Martin) ${ }^{52}$ et eut deux fils connus : Pierre et Jean. Ce dernier portait tantôt un titre tantôt non ${ }^{53}$ : lorsqu'il avouait en 1440 la Salle du Vieux-Pont à Alixd'Estouteville, il était écuyer ${ }^{54}$. Toutefois, lorsque cette dernière dénombrait la seigneurie du Bouchet au comte, elle ne mentionnait pas l'armigérat de cet arrière-vassal ${ }^{55}$. Cette diversité de titres, ces dénominations hésitantes, nous permettraient de dresser le portrait d'un lignage en voie d'anoblissement, à mi-chemin entre chevalerie et bourgeoisie. D'ailleurs, le manque d'enthousiasme d'Estouteville à reconnaître l'armégirat de son " home de foy " ne prouvait-il pas le réflexe nobiliaire ou la réaction aristocratique (si souvent décrits) face à des parvenus un peu trop pressés? En fait, les rédacteurs des actes étaient différents de même que les enjeux sous-jacents à la rédaction. Jehan Beauxoncles était qualifié de "noble homme et monsieur " (voilà qui fleure bon la chevalerie...) par son " home " Jehan de Beauvoir l'aîné : de cette constatation, nous pouvons certes déduire une trace d'ascension sociale. Pouvons non aussi envisager cet élément comme un révélateur du lien mental qui associait fortement seigneur et état de noblesse ${ }^{56}$ ? Construisait-il ainsi l'identité sociale du vassal? La mention écrite d'un titre nobiliaire pouvait-elle favoriser ou consolider les prétentions à l'anoblissement? En fait, en posant cette question, nous sommes quelque peu victime de la vision de la noblesse que pouvaient avoir les généalogistes royaux du XVII ${ }^{\mathrm{e}}$ siècle, tels d'Hozier. La consultation de ses " dossiers bleus " ou de ses "Pièces Originales ${ }^{57}$ " illustre à merveille la démarche de l'auteur de l'Armorial : la recherche éperdue, minutieuse, la traque même, du moindre indice de noblesse (écuyer, chevalier, dame ou demoiselle voire monseigneur ou seigneur)... L'analyse cri-

50. Bibliothèque nationale : $\mathrm{ms}$ fr $25265, \mathrm{n}^{\circ} 114$ ("Saichent en nostre cour de Vendosme, Jehan Beauxoncles demourant à Vendosme "). Bibliothèque nationale : Pièces originales $\mathrm{n}^{\circ} 6$ du cabinet d'Hozier, PO 257 (" De vous noble homme monsieur Jehan Beauxoncles, seigneur de Fay, Je Jehan de Beauvoir l'aîné... ").

51. Arch. dép. de Loir-et-Cher, G 239, 1425.

52. "Item la dicte Guillemecte de Saint Martin fut conjoincte par mariage avec feu messire Jacques Beauxoncles en son vivant chevalier duquel mariage furent nés feu Jehan Beauxoncles dernier seigneur du Plaissis Saint Martin et ledit Pierre Beauxoncles ". Bibliothèque nationale : Pièces originales $n^{\circ} 29$ du cabinet d'Hozier, $\mathrm{PO} \mathrm{n}^{\circ} 257$.

53. «De vous Louis de Bourbon comte de Vendosme, Je Jehan Beauxoncles tiens à foy et homage lige les choses dont je suis en vostre foy et homage lige ". Arch. nat., P 627, $\mathrm{n}^{\circ} 68,1435$.

54. "De vous, noble dame, je Jehan Beauxoncles écuyer tiens... ", Arch. nat., P 611, $\mathrm{n}^{\circ} 37,1440$.

55. "Jehan Beauxoncles est mon home de foy simple et un roucin de service pour son lieu appelé la Salle de Vieux-Pont ", Arch. nat., P 611, n 30, 1445.

56. Dit autrement, " état seigneurial " et " état nobiliaire " pouvaient être associés dans une même représentation sociale de l'aristocratie (quasiment assimilable aux mythes et croyances des sociétés traditionnelles), largement partagée par les hommes du XV $\mathrm{V}^{\mathrm{e}}$ siècle : JoDELET, Denise (dir.), Les Représentations sociales, Sociologie d'aujourd'hui, PUF, Paris, 1989, p. 18.

57. Bibliothèque nationale, cabinet des Titres. 
tique de l'acte n'était pas de rigueur alors que l'objectif était de légitimer, a posteriori, la noblesse d'une famille quelque peu embarrassée par les injonctions du Roi Soleil. Cette méthode, trop souvent reprise par la suite, nous empêche peut-être de comprendre la spécificité bas médiévale. L'aveu n'était pas destiné à justifier une situation sociale ${ }^{58}$ : tout au plus un droit sur la terre. Ainsi, la cérémonie des assises, la présence aux montres du duc d'Anjou, ou les aspects symboliques de la résidence seigneuriale comptaient probablement davantage que la mention ou l'absence d'un titre nobiliaire qui, du reste, n'était pas figé.

Nous l'avons précisé plus haut, le corpus des aveux et dénombrements ne forme pas un bloc monolithique : les auteurs de l'acte notamment variaient ("l'home de foy ", son procureur, ou bien la chancellerie du comté dunois ou blésois), de même que la qualification du vassal. Il faut attendre 1484 pour connaître le premier feudataire à se dénommer " bourgeois demeurant à Vendôme ${ }^{59}$ "; 1487 pour connaître le premier « marchand ${ }^{60}$ "; 1477 pour le premier licencié en loi ${ }^{61}$. Ces ascensions sociales, concentrées dans un laps de temps assez court, seraient-elles liées au rôle essentiel que jouèrent de nombreux " bourgeois conquérants " durant la reconstruction du royaume (économique et juridique notamment)? Nous pensons qu'elles sont davantage le révélateur d'un phénomène bien antérieur. En effet, les bourgeois étaient présents dans le monde des " homes de foy " depuis plus d'un siècle. Le Livre des fiefs en connaissait trois : Colin de Boisraoul bourgeois de Vendôme; Bouchard l'Usurier et Jehan Boien tous deux bourgeois du Mans ${ }^{62}$. De plus, bien avant 1484, on pouvait être veuve ou fils de bourgeois sans pour autant porter le titre ${ }^{63}$. D'une manière générale, nous constatons que les vassaux du comte portaient assez peu de qualificatifs à la fin du XIV ${ }^{\mathrm{e}}$ siècle.

\footnotetext{
58. Les quelques " preuves de noblesse " consultées mentionnent assez peu le recours aux " vieils escripts ". Il était fait davantage mention du mode de vie, des relations de parenté... En cela, nous rejoignons les constatations de Michel Nassiet qui note le développement, à partir du début du xvie siècle seulement, du recours à la preuve écrite dans le discours généalogique (annonçant ainsi la réforme de 1666 qui rendait obligatoire la preuve écrite pour prouver la noblesse) : NASSIET, Michel, «La généalogie entre discours oral et écrit ( $\mathrm{Xv}^{\mathrm{e}}-\mathrm{XVI}^{\mathrm{e}}$ siècles) ", BARThelemy, Tiphaine, Pingautd, Marie-Claude, La Généalogie entre science et passion, Paris, Éditions du Comité des travaux historiques et scientifiques, 1997, p. 207-220.

59. " Je Michel Guinebault, bourgeois demourant à Vendosme confesse... ". Arch. nat., $\mathrm{P} 625, \mathrm{n}^{\circ} 22,1484$.

60. "De vous, comte de Vendosmois, Epernon et Remaillart, Je Thomin Bérandeau, marchand demourant à Vendosme tiens... ", Arch. nat., P 627, n 101, 1487.

61. "De vous, Je Michel le Tillier dit Fleury, licencié en lois, fils aîné et principal héritier de feu Mathurin le Tillier dit Fleury mon père... ", Arch. nat., P 623, n 90, 1477.

62. Arch. nat., P 976, 1355.

63. "Je Gilon famme jadis de feu Pierre de Villebresme escuyer jadis bourgeois de Blois ", Arch. nat., P 611, n 52, 1370. " Je Jehan Gaillard, fils de feu Jehan Gaillard jadis bourgeois de Blois ", Arch. nat., P 611, n 51, 1437.
} 
Tableau 1 - Le port d'un qualificatif dans les aveux directs : écuyer, chevalier, demoiselle, seigneur de, dame de, bourgeois, marchand, juriste, artisan, habitants de, officier

\begin{tabular}{|c|c|c|}
\hline Décennies / aveux & $\begin{array}{c}\text { Feudataires } \\
\text { qualifiés }\end{array}$ & $\begin{array}{c}\text { Feudataires } \\
\text { non qualifiés }\end{array}$ \\
\hline $1350-1390$ / 29 & $27,5 \%$ & $72,5 \%$ \\
$1390-1400$ / 27 & $44,5 \%$ & $55,5 \%$ \\
$1400-1409$ / 36 & $52,8 \%$ & $47,2 \%$ \\
$1410-1449$ / 36 & $58,5 \%$ & $41,5 \%$ \\
$1450-1479$ / 36 & $58,5 \%$ & $41,5 \%$ \\
$1480-1489 / 36$ & $77,8 \%$ & $22,2 \%$ \\
$1490-1499 / 15$ & $66,7 \%$ & $33,3 \%$ \\
$1500-1509 / 45$ & $64,5 \%$ & $35,5 \%$ \\
$1510-1519 / 46$ & $74,0 \%$ & $26,0 \%$ \\
$1520-1539 / 35$ & $78,0 \%$ & $22,0 \%$ \\
$1540-1549 / 31$ & $77,5 \%$ & $22,5 \%$ \\
\hline
\end{tabular}

Tableau 2 - Le port d'un qualificatif dans le Livre des fiefs : écuyer, chevalier, demoiselle, seigneur de, dame de, bourgeois, marchand, artisan, juriste, habitant de, officier

\begin{tabular}{|c|c|c|}
\hline Livre des fiefs & $\begin{array}{c}\text { Feudataires } \\
\text { qualifiés }\end{array}$ & $\begin{array}{c}\text { Feudataires } \\
\text { non qualifiés }\end{array}$ \\
\hline 1355 (81 feudataires) & $75 \%$ & $25 \%$ \\
\hline
\end{tabular}

Nous pourrions invoquer les progrès de l'administration ou de la pratique de l'écrit, qui justifieraient la plus grande précision des actes. Une remarque s'impose toutefois. Si les vassaux hésitaient à se qualifier, les scribes comtaux n'avaient pas autant de scrupules. Jacquet du Pont, rédacteur du Livre des fiefs, retranscrit-il fidèlement les aveux qu'il utilisa ${ }^{64}$ ou bien ajouta-t-il quelques titres, contribuant ainsi à représenter et à construire la société qu'il voyait vivre face à lui? Le Tableau 2 nous inciterait à privilégier la seconde hypothèse. La remarque serait également valable pour les officiers des cours de Châteaudun ou de Blois, qui rédigèrent à la demande de quelques feudataires les aveux rendus au comte de Vendôme ${ }^{65}$. D'une part, ils nous permettent de connaître des juristes ou bourgeois « homes de foy " avant la fin du Xve siècle ${ }^{66}$. D'autre part, ils n'hé-

64. "Selon ce que ledit jacquet les a trouvés et verefiés par enquestes par monstrées et informations faittes o les anciens et savanz du pais et par aucuns advous anciens, avec les quelx il en a fait concordance ", Arch. nat., P 976, 1355.

65. "Devant nous, justice de la chastellenie de Chasteaudun est comparu le doyen et chapitre de l'esglise collégiale de Saint André qui ont asvoué tenir en la conté, pays et terre de Vendosme... ", Arch. nat., P 639, n 33, 1436.

66. Honorable homme et maître Guillaume des Moulins, licencié en lois, avouait ses métairies de Villebadain et Mihaudouin en 1457 (Arch. nat., P 623, n 94, 1457). Pierre 
sitent pas à les qualifier de " noble homme ${ }^{67}$ " alors que ce titre fut employé assez rarement et très tardivement par les vassaux eux mêmes ${ }^{68}$.

La faiblesse du nombre de qualification dans les aveux directs interpelle le lecteur du tableau 1. Le premier réflexe, nous l'avons vu, serait de faire de ces non-qualifiés des roturiers, parfois en voie d'anoblissement. En 1397, Pierre de Lespine déclarait sa pêcherie du Loir ${ }^{69}$. Fils d'un anobli royal, il servait dans la compagnie du comte de la Marche (également comte de Vendôme) en tant qu'écuyer quelques années auparavant ${ }^{70}$. Il faut attendre 1409 pour qu'il accède à l'armigérat dans un aveu ${ }^{71}$, grâce peut-être à la " patine du temps " chère à Dominique Barthélemy. Les Malon, Beauvoir ou Savigne vérifieraient l'hypothèse : l'étude de leur cas tendrait à prouver qu'un lien unissait les non titrés à la notabilité urbaine. Toutefois, transformer cette hypothèse de travail en corrélation se révèle vite dangereux. Pierre Belon était simplement seigneur de la Boissière en 1371 avant de devenir écuyer en $1397^{72}$. Bourgeois d'après R. de SaintVenant ${ }^{73}$ ou D. Barthélemy ${ }^{74}$, il appartenait plus sûrement à une branche cadette du puissant lignage des seigneurs de Lorges, connus depuis le

Lesbahy, marchand bourgeois d'Orléans, tenait en fief des terres à Périgny en 1469 (Arch. nat., P 623, n ${ }^{\circ}$ 28, 1469).

67. Noble homme Jehan de Saint-Martin, écuyer et fils de feu Odet de Saint-Martin, était seigneur d'une métairie aux Hayes en 1405 (P 652, n 1405).

68. La première mention n'est pas antérieure à 1516 : à cette date, noble homme Jehan de Cuigy, licencié en lois, conseiller et procureur général du roi en Touraine, avouait sa terre et seigneurie de la Quesnière en Villeromain (Arch. nat. : $\mathrm{P} 625, \mathrm{n}^{\circ} 8,1516$ ).

69. Arch. nat., $\mathrm{P} 627, \mathrm{n}^{\circ}$ 52, 1397.

70. « Nous vous envoyons attachiée sous nostre scel commun de la mareschauciée la monstrée de Pierre de Lespine, escuier et de huit autres escuiers de sa chambre reçue à Orléans le 2 septembre 1386 pour servir aux gaiges du roi en cestes présentes guerres et en ce présent voyaige d'Angleterre en la compagnie de monsieur le comte de la Marche "; "Saichent tous que je Pierre de Lespine escuier confesse avoir eu et reçu de Jehan le Flament trésorier des guerres du roi la somme de 67 livres 10 sous. " Bibliothèque nationale : Pierres originales $\mathrm{n}^{\circ} 5$ et 6 du cabinet d'Hozier, PO 1697.

71. Arch. nat., $\mathrm{P} 627, \mathrm{n}^{\circ}$ 53, 1409.

72. Arch. nat., $\mathrm{P} 601, \mathrm{n}^{\circ} 56,1371 ; \mathrm{n}^{\circ} 59,1397$.

73. En 1364, l'aveu de la Maison-Dieu spécifiait que « Dame Jehanne de la Brosse tient de Céans à Foy et hommage et un roucin de service les fiefs et arrière-fiefs qui s'ensuivent : Foy et hommage à elle due par Geuffroy de Belon pour sa métairie des Murats (Villeromain)" (Bibliothèque municipale de Vendôme, ms 285, 1364). Or, quelques années plus tard, Geuffroy Belon vendait à Macé Copelet et Jehan Malon sa part de la dite métairie (Bibliothèque municipale de Vendôme, ms 285, 1367). Il n'en fallut pas plus à R. de Saint-Venant qui fit des Belon de bourgeois de Blois : Saint-Venant (R. de), op. cit., II, p. 470. Il était probablement trompé par le fait que les Murats relevaient de la Dame du Quartier de Blois. Toutefois, dans le même temps, il notait la présence de Jehan Belon, capitaine du château de Vendôme en 1328, lieutenant de Jehan de Lisle et capitaine pour le roi en Poitou. En 1363, il était maréchal d'Amaury de Craon, lieutenant du roi en Anjou, Maine et Touraine : Saint-Venant (R. de), op. cit., I, p. 109.

74. Dominique Barthélemy notait que Jehanne de la Boissière « finit par recueillir le tout et, avec son mari Jehan de Boisgarnier, par vendre les trois fiefs avant 1376 aux Belon (Villiers) et aux Malon (Villetrun) - c'est à dire à des bourgeois de Vendôme ": BARTHELEMY, Dominique, op. cit., p. 863 et 982 . 
$\mathrm{XIII}^{\mathrm{e}}$ siècle ${ }^{75}$. Nous pourrions dire la même chose des Vaulevrier ${ }^{76}$, et surtout des Marray, Ternay et Sainte-Maure ${ }^{77}$. Faire de la présence obligatoire de la chevalerie ou de l'armigérat pour déduire la noblesse d'un individu serait une erreur : cette démarche postulerait la supériorité du titre sur le mode de vie. Elle ceindrait en deux groupes bien distincts ceux qui jouissaient pleinement du statut de vassal : un statut revivifié périodiquement et publiquement lors des assises du Vendômois, par un rite dont nous pensons qu'il était pour une part constitutif de "l'état de noblesse ${ }^{78}$ ". Pierre Bourdieu a souvent mis en garde contre la tentation de "découvrir dans le discours même le principe de l'efficacité de la parole " en indiquant que " le langage, en fait, représentait, manifestait, symbolisait le pouvoir de l'orateur ${ }^{79}$ " : dit autrement, jusque dans la seconde moitié du Xve siècle, qu'un vassal se proclame bourgeois ou écuyer était-il essentiel? Etait-ce plus important que d'être présent, tout simplement, durant les assises, prêtant serment ou rendant dénombrement? Etait-ce plus important que d'avoir accès à cette parcelle du " langage légitime " qu'était l'hommage?

L'incompatibilité jusqu'à la fin du Xve siècle (tout au moins lexicale et documentaire) entre vassalité et bourgeoisie urbaine semble illustrer l'image d'un monde où service vassalique et état de noblesse étaient liés (et excluaient par là même la notabilité urbaine). Une image défendue par les vassaux eux-mêmes qui s'attachaient à rendre " l'acte acceptable ${ }^{80}$ " en n'y

75. Dès 1293 Harmé Belon, chevalier et seigneur de Lorges (en Beauce), vendait au comte de Vendôme 30 livres assises sur les rentes de Vendôme qu'il avait héritées de sa femme (Arch. nat., Q 1443, 1293). On retrouve des Belon seigneurs de Lorges au XIII ${ }^{\mathrm{e}}$ siècle. La terre passa par la suite aux Le Jay, puis aux Montgomery (l'héritière Le Jay épousait en secondes noces Robert de Montgomery en 1481) : MARQUENET, Georges, " Le château de Lorges ", FALLOT, Joëlle (dir.), Patrimoine dans votre commune : Lorges, CDPA, Blois, 1994.

76. " De vous très excellent et puissant prince et seigneur monsieur le comte de la Marche, de Vendôme et de Castres, Je Pasquier de Vaulevrier tiens... ", Arch. nat., P 601, $\mathrm{n}^{\circ} 10,1374$. Or, dès 1314, Pierre de Vaulevrier, écuyer, vendait des biens à Gervaise Guerteau qui devait payer le franc-fief aux commissaires du duc d'Anjou. Bibliothèque nationale : $\mathrm{ms}$ fr 8736 .

77. "Je Jehan de Marray tiens à cause de ma femme... ", Arch. nat., P 657, n 67, 1375. " Je Odet de Turné confesse être home de foy ", Arch. nat., P 657, n 30, 1399. "Je Jehan bastard de Sainte-Maure tiens ma mestairie... ", P 625, n 7, 1389. Or, l'ancienneté de ces trois familles chevaleresques remontait au XIe siècle : BARTHELEMY, Dominique, op. cit., p. 721 et 964 .

78. Nous avons indiqué plus haut que l'hommage public transformait la personne consacrée et la représentation que s'en faisait les autres. Peut-on imaginer également qu'elle modifiait aussi la représentation que le vassal nouvellement investi se faisait de lui même et les comportements qu'il se croyait désormais tenue d'adopter pour se conformer à cette représentation? En d'autres termes, quelle était la part de l'obligation et du choix dans l'accession au "mode de vie nobiliaire "?

79. Bourdieu, Pierre, Ce que parler veut dire..., op. cit.

80. " Il s'agit, du moins en théorie, de rendre l'acte acceptable, ce qui met en jeu des mouvements complexes d'interactions entre ce que l'on peut souhaiter transmettre, du producteur au récepteur donc, et l'idée que l'on peut avoir de ce qui doit être transmis et comment, du producteur au producteur donc, et finalement aussi du récepteur potentiel au producteur ": BARRET, Sébastien, "Stéréotype et inventivité dans les préambules d'actes médiévaux ", Zimmermann, Michel, Auctor et auctoritas..., op. cit., p. 320. 
indiquant pas une mention qui serait contraire à cette vision du monde; qui jugeaient inutiles (redondants?) de s'intituler eux-mêmes « nobles »? Les historiens ont souvent pensé que le titre de "noble homme " était porté par des roturiers en mal d'anoblissement (ou ennoblis), à qui étaient refusés le port plus prestigieux de l'armigérat ou de la noblesse. Loin de prouver la noblesse, il en serait davantage une revendication. Si le corpus vendômois ne permet pas véritablement de trancher ${ }^{81}$, il autorise deux constations : d'une part, les " nobles " (hommes, seigneurs...) n'apparaissent en nombre qu'à la fin du $\mathrm{XV}^{\mathrm{e}}$ siècle contribuant ainsi à donner l'impression d'une véritable « invention de la noblesse ${ }^{82}$ ». D'autre part, les vassaux ne se titraient quasiment jamais ainsi ${ }^{83}$. Ils se déclaraient " fils ou veuve de noble homme " ou bien étaient qualifiés de " nobles " par leurs procureurs ou les cours châtelaines dans le cas d'aveux indirects ${ }^{84}$. "Écuyer ", " chevalier ", " demoiselle " ou " dame " prouvaient plus sûrement l'appartenance à l'aristocratie. Les hommes de foi portaient assez fréquemment ces titres au XIVe et XV siècles : au fil du temps, ils furent de plus en plus couplés avec celui de « seigneur " dont la révélation rapide et imposante après 1480 pose un certain nombre de questions.

Tableau 3 - L'emploi des titres nobiliaires (écuyer, chevalier, demoiselle, dame) et seigneuriaux

\begin{tabular}{|c|c|c|c|}
\hline Décennies & Aveux & Titre nobiliaire & $\begin{array}{c}\text { seigneur de, } \\
\text { dame de }\end{array}$ \\
\hline $1350-1390$ & 29 & $24,0 \%$ & $10,3 \%$ \\
$1390-1400$ & 27 & $33,3 \%$ & $3,7 \%$ \\
$1400-1409$ & 36 & $44,5 \%$ & $8,3 \%$ \\
$1410-1449$ & 36 & $52,8 \%$ & $27,8 \%$ \\
$1450-1479$ & 36 & $33,3 \%$ & $22,2 \%$ \\
$1480-1489$ & 36 & $61,0 \%$ & $47,2 \%$ \\
$1490-1499$ & 15 & $33,3 \%$ & $6,7 \%$ \\
$1500-1509$ & 45 & $24,5 \%$ & $22,2 \%$ \\
$1510-1519$ & 46 & $26,0 \%$ & $30,4 \%$ \\
$1520-1539$ & 35 & $45,7 \%$ & $31,4 \%$ \\
$1540-1549$ & 31 & $29,0 \%$ & $38,7 \%$ \\
\hline
\end{tabular}

81. Des chevaliers d'anciens lignages sont dits " nobles hommes ", comme Jehan de Saint-Martin (Arch. nat., P 652, 1405); mais le titre est également porté par des anoblis, comme Guillaume Malon (Arch. nat., $\mathrm{P} 657, \mathrm{n}^{\circ} 39,1486$ ).

82. Morsel, Joseph, "L'invention de la noblesse en Haute-Allemagne à la fin du Moyen Âge. Contribution à l'étude de la sociogenèse de la noblesse médiévale ", PAvIoT, Jacques, VERGER, Jacques (dir.), Guerre, pouvoir et noblesse au Moyen Âge. Mélanges en l'honneur de Philippe Contamine, Paris, Presses de l'Université de Paris-Sorbonne, 2000, p. 533-546. Morsel, Joseph, La Noblesse contre le prince. L'espace social des Thüngen à la fin du Moyen Âge (Franconie, ca. 1250-1525), Stuttgart, "Beihefte der Francia, 49 », 2000.

83. Le seul contre-exemple est celui de " noble homme maître Jehan de Cuigy, licencié en lois, conseiller et procureur général du roi en Touraine ", Arch. nat., P 625, n 8, 1516

84. "Jehan Georget chastellain garde de justice en la cour de vendosme en l'absence de Guyon Farineau bailli de Vendosme a comparu devans nous noble homme messire Jehan de Brée chevalier, seigneur de Chasle ". Arch. nat., P 657, n 19, 1429. 


\section{Le fief, la seigneurie : une ou plusieurs réalités médiévales?}

Il semble bien que derrière ces deux termes se cachent des réalités multiformes et qui évoluent pendant les deux derniers siècles du Moyen Âge. Dans vingt-quatre dénombrements seulement, les vassaux avouent une seigneurie, assez tardivement d'ailleurs (la première mention date de $1454^{85}$ ). Dans la plupart des cas, le dénombrement se résumait en une liste de terres, de cens et de rentes, autour d'un lieu d'habitation (l'habergement ou la métairie) sans que se dégage l'impression d'une unité : peut-être devrait-on d'ailleurs parler d'inventaire et non de liste ${ }^{86}$. La seigneurie n'était alors pas pensée comme un tout car elle n'était pas nommée comme un tout. Les aveux (sans dénombrements) de 1484 et 1516 favorisèrent une véritable révolution lexicale. La seigneurie et le fief furent alors brutalement révélés. Les différentes composantes du bien hommagé étaient ainsi englobées dans une réalité nouvelle : grâce à l'aveu, l'unité de la chose, par delà ses occurrences diverses, était posée. Elle était conçue comme un seul et unique objet ${ }^{87}$. Cette prise de conscience de la seigneurie et du fief, grâce aux aveux, eut des répercussions dans les dénombrements suivants : ce fut le cas pour Teillé (Espéreuse). En 1406, Jehan de Noce avouait et dénombrait son " habergement de Teillé en maisons, four, court, courtils ${ }^{88}$... ". S'ensuivait un inventaire fourni, énumérant les terres, cens, bois, métairies, arrière-fiefs déclarés par le vassal. Le modèle fut repris par la suite, en 1442 et $1498^{89}$. L'aveu de 1483 concernait la " terre, fie et seigneurie du lieu de Tellé " non autrement détaillé ${ }^{90}$. Or ce sont des termes similaires qui furent repris dans un dénombrement de 1515, qui connaissait désormais « ledit lieu, fief, terre et seigneurie de Teillay ${ }^{91}$ ". Les aveux de 1484 et 1516 jouèrent donc probablement un rôle essentiel dans la prise de conscience d'une nouvelle réalité ${ }^{92}$. Deux questions mériteraient réflexion. L'objet décrit changeait-il également de nature, acquérant par exemple une nouvelle cohérence? De plus, cette " nouvelle réalité " était-elle aussi nouvelle que nous voulons bien le croire? Ne venait-elle pas compenser les conséquences du dénombrement

85. Pierre Bouchier dénombrait alors son " fie et seigneurie de Chappedasne ", Arch. nat., $\mathrm{P} 625, \mathrm{n}^{\circ} 35,1454$.

86. GoODY, Jack, La Raison graphique, op. cit., p. 140 et suivantes.

87. ERALY, Alain, L'Expression et la représentation. Une théorie sociale de la communication, Logiques sociales, Paris, L'Harmattan, 2000, p. 216 et suivantes.

88. Arch. nat., P 625, n $^{\circ} 59,1406$.

89. Arch. nat., P 625, $\mathrm{n}^{\circ} 58$ et $61,1442,1448$.

90. Arch. nat., P 625, $\mathrm{n}^{\circ} 56,1483$.

91. Arch. nat., P 625, n 50, 1515.

92. Parallèlement il semble (mais cela devrait être vérifié systématiquement) que le dénombrement prend davantage l'aspect d'une liste : les item annonçant la future description ("Item ma tousche de bois de hute futays contenant 24 arpents; Item 15 arpents de bois menus... ") sont écrits en caractères gras, impliquant ainsi " un certain agencement matériel, une certaine disposition spatiale facilitant la lecture grâce à la mise en ordre des articles par leur numérotation ": Goody, Jack, La Raison graphique, op. cit., p. 140 et suivantes. 
écrit qui accentuait la discontinuité du bien déclaré en lui conférant une dimension spatiale et visuelle sur le parchemin?

Les mêmes remarques s'appliqueraient au fief. La seconde moitié du $\mathrm{XIV}^{\mathrm{e}}$ siècle entendit le fief comme une mouvance féodale (c'est-à-dire présence d'arrière-vassaux), opposée au domaine qui était exploité directement par le seigneur, loué ou bien accensé. Alors que Ysabeau de SaintAmand avouait en 1371 " les choses dont je suis en vostre foy et homage tant fies comme domaines ", Guillaume de Saint-Amand dénombrait quelques années plus tard " une métairie et la grange que j'ai à la ToucheChenard... Item j'avoue tenir de vous une fie que les hoirs feu monsieur Guillaume du Plessis doivent tenir de moi à foy et homage ${ }^{93}$ ". En fait, derrière la règle, les exceptions sont nombreuses. "Le fie du Boile " (Pezou) ${ }^{94}$, le « fie Tafforeau " (Lunay) ${ }^{95}$, le " fie Possonnière " (Coutures) ${ }^{96}$ ne comportaient pas d'arrière-vassaux mais une censive dont l'importance semblait d'ailleurs être liée au terme de fief : " Je Phelippe Marques à cause de Jehanne de la Vallée ma femme avoue les choses dont je suis vostre home de foi simple, et premièrement, mon fie appelé le fie Possonnière à Coustures assavoir dix sous que les hoirs..." (suit une liste de censitaires, cens et biens accensés avec les confronts de parcelles) ${ }^{97}$. L'exemple du Boile irait également dans ce sens : "Un petit fie appelé le fie du Boile à ventes et reliefs qui soulloit valoir anciennement 25 sous mais depuis n'a connaissance que de 12 sous 6 deniers ${ }^{98}$. " L'image d'une frontière très floue entre censive et arrière-fief est illustrée par la présence d'un arrière-vassal dans un dénombrement de 1440, aucunement dégagé des censitaires de la Possonnière qui l'entourent ${ }^{99}$. Les années 1480 marquent une véritable "révélation du fief " (avec plus de 80 \% des mentions du corpus vendômois). Le terme, très souvent associé à celui de seigneurie, se répandit pour donner une unité nouvelle au bien hommagé. Né dans les aveux de 1484, il concerna également très vite les dénombrements. Ainsi, l'origine du fief (tel qu'il est défini dans les manuels d'histoire) ne semble pas devoir être placée avant la fin du $\mathrm{XV}^{\mathrm{e}}$ siècle.

\section{4-1516 : une " ré-formation " des structures mentales?}

Les innovations lexicales des années 1484 et 1516 peuvent être expliquées par la typologie de l'acte. Celui-ci, appelé simplement " étiquette ${ }^{100}$ "

93. Arch. nat., $\mathrm{P} 627, \mathrm{n}^{\circ} 88$ et 90,1400 et 1371.

94. Arch. nat., $\mathrm{P} 625, \mathrm{n}^{\circ} 30,26,24 ; 1375,1396,1399$.

95. Arch. nat., $\mathrm{P} 657, \mathrm{n}^{\circ} 7,8 ; 1399,1456$.

96. Arch. nat., $\mathrm{P} 657, \mathrm{n}^{\circ} 85,1440$.

97. Arch. nat., $\mathrm{P} 657, \mathrm{n}^{\circ} 85,1440$.

98. Arch. nat., $\mathrm{P} 625, \mathrm{n}^{\circ}$ 25, 1460.

99. «Hoirs feu Jehan Bouquereau pour leur cloux de vigne d'Aubemare foy et homage et 16 deniers de service ", $\mathrm{P} 657, \mathrm{n}^{\circ} 85,1440$.

100. «Signée ceste présente étiquette de ma main et à ma requeste du seing manuel de Jehan Trépereau tabellion de Vandosme ", Arch. nat., P 625, n 17, 1516. 
était alors bien différent des précédents. Moins solennel ${ }^{101}$, rédigé sur papier non sur parchemin, relativement court (généralement autour d'une centaine de mots), l'aveu sans dénombrement de la fin du $\mathrm{Xv}^{\mathrm{e}}$ et du $\mathrm{XVI}^{\mathrm{e}}$ siècles était moins officiel : or, les variations de la forme d'un discours, par ailleurs probablement contrôlé, surveillé, dépendent pour une part " de la tension objective du marché ${ }^{102}$ ", c'est-à-dire du degré d'officialité de la situation. Moins celui ci était élevé, plus celles là étaient possibles. D'autre part, la disparition de l'inventaire rendait son unité au bien déclaré, permettant ainsi " l'invention de la seigneurie ». Ces changements lexicaux ne doivent pas être minorés car avec ce nouveau langage imposé, était également imposée " une formation ou ré-formation des structures mentales " (qui transformaient à leur tour la réalité sociale) : il ne s'agissait pas simplement de communiquer mais de faire reconnaître un "nouveau discours d'autorité, avec son nouveau vocabulaire politique, ses termes d'adresse et de référence, sa représentation du monde social qu'il véhicule ${ }^{103}$ ". En modifiant la représentation de la vassalité vendômoise, on contribuait également à modifier le monde des vassaux ${ }^{104}$. Le problème du contrôle de ces " visions du monde " se pose donc avec beaucoup d'acuité : G. Fournier se demandait lors du colloque de Pont-à-Mousson consacré à la maison forte si c'était la pensée du seigneur ou du vassal qui prédominait dans les aveux et dénombrements. En Vendômois, qui avait une autorité symbolique assez forte, c'est-à-dire un pouvoir socialement reconnu par tous d'imposer une nouvelle vision du monde social, si ce n'est le comte de Vendôme conforté par son nouveau prestige (vassal direct du roi puis duc en 1516?). La similitude des aveux, malgré des rédacteurs différents (notaires vendômois, tabellions du Mans ou de Tours, vassaux eux mêmes) nous incite à envisager l'existence d'un formulaire, rédigé par la chancellerie comtale ${ }^{105}$.

Jusqu'à la fin du $\mathrm{XV}^{\mathrm{e}}$ siècle, "l'état de noblesse " n'était pas affaire de titre. Prisonnier d'une vision dix-septièmiste de la société, nous avons traqué, comme d'Hozier, les références à la chevalerie ou à l'armégirat, oubliant les allusions faites aux montres ${ }^{106}$, à la participation aux rites vas-

101. Il ne semble pas avoir été rendu à l'occasion d'assises (quoi qu'il en soit, aucune mention ne semble aller en ce sens). Il n'était pas scellé à la différence des dénombrements.

102. BourdiEu, Pierre, Ce que parler veut dire..., op. cit., p. 103-119 (« Le langage autorisé : les conditions sociales de l'efficacité du discours rituel ").

103. Ibid., p. 31.

104. Peut-être doit-on davantage parler de plusieurs " mondes des vassaux vendômois ", c'est-à-dire de plusieurs visions, concurrentes entre elles : ces mondes sont une représentation sociale qui varie si le sujet est le vassal, le comte, une cour de justice...

105. En effet, il n'existait pas de modèle ancien accessible pour rédiger les aveux de 1484 et 1516 (les hommages écrits de la fin du XIVe siècle s'en approchaient mais n'amorçaient même pas la description du bien déclaré : " ce que je tiens en votre foi et homage tant fie comme domaine" $"$.

106. "Ce sont les noms des gens nobles des ressorts et seigneuries de Baugé qui se sont comparus aux monstres dudit lieu de Beaugé, le jeudi 27 décembre 1470 " (le comté de 
saliques (hommage et remise du dénombrement), au choix du lieu de sépulture $^{107}$, au symbolisme de la résidence seigneuriale ${ }^{108}$ et des armoiries... Ces divers éléments étaient autant de preuves d'une appartenance à un groupe particulier, d'une élite qui ne se dénommait pas noble, qui ne partageait pas les mêmes titres ${ }^{109}$, mais dont on pressent une solidarité de mode de vie.

À partir de la seconde moitié du Xve siècle, les vassaux furent mieux connus : en 1484 et 1516, leur qualification prenait souvent plus d'importance que l'aveu de ce qu'il était désormais convenu d'appeler « fief et seigneurie ${ }^{110}$ ». En outre, un véritable système de titre, plus rigoureux, se mit en place, assurant une nouvelle cohérence à la société pensée par les aveux et dénombrements ${ }^{111}$. La vision du comte, palpable dès le Livre des fiefs dans lequel on sentait l'autorité publique pressée d'octroyer une identité moins floue à chaque vassal, mit quasiment un siècle et demi à triompher, aidée en cela par les progrès de l'écrit qui permettait de formaliser plus nettement la place de chacun. Ainsi, le statut de vassal devenait moins important que la référence à la noblesse, à la bourgeoisie ou à l'office judiciaire... L'écrit devenu véritable preuve n'affirmait plus seulement un droit sur la terre et les hommes mais également une noblesse (les écrites généalogiques suivirent les mêmes chemins), davantage contrôlée par l'autorité comtale : ainsi triomphait sa vision d'un monde mieux défini, mieux surveillé et peut-être plus fermé. La participation aux rites ne permettait plus à elle seule les ascensions au sein d'un groupe (celui des vassaux) désormais fractionné. Une sorte d'ordre était affirmé avec force par les aveux et dénombrements, qui mettait fin aux bouleversements sociaux de la guerre

Vendôme dépendait jusqu'en 1484 du ressort de Baugé). Bibliothèque nationale : ms. fr. $11864,1470$.

107. Les élites vendômoises avaient le choix entre la collégiale urbaine Saint-Georges de Vendôme (le "Saint-Denis vendômois " qui accueillait également les dépouilles des comtes) et l'abbaye de la Trinité, au profil plus rural et aristocratique.

108. L'étude du manoir de la Secrétainerie (Pezou) devrait être riche d'enseignements. Il avait été bâti durant le XIve siècle par les Beauxoncles qui firent sculpter leurs armoiries sur le manteau de la grande cheminée : SAINT-VENANT, Raoul Barré de, op. cit., III, p. 372 .

109. D. Barthélemy a avancé l'hypothèse, pour une période antérieure, que l'ensemble du monde paysan pouvait être " en servitude " alors qu'une minorité seulement était qualifiée de serfs (BARThelemy, Dominique, La Mutation de l'an Mil a-t-elle eu lieu?, Paris, Fayard, 1997) : ainsi, la présence d'un titre nobiliaire pourrait ne pas remettre en cause l'unité fondamentale du monde des vassaux du comte.

110. " Noble homme maistre Jehan de Cuigy, licencié en lois, conseiller et procureur général du roi au bailliage de Touraine confesse estre home de foi de Charles duc de Vendosme pair de France pour raison de ma terre et seigneurie de la Cousinière à foi et homage lige et quinze jours de garde au chastel de vandosme ", Arch. nat., P 625, $\mathrm{n}^{\circ} 6,1516$.

111. Nous avons relevé les mentions de veuves dans les aveux et dénombrements. Entre 1350 et 1500 , il y avait cohérence entre le qualificatif de la veuve et celle de son époux dans $40 \%$ des cas (une demoiselle veuve d'un écuyer; une dame veuve d'un noble homme; une honnête femme veuve d'un maître...). Entre 1500 et 1549, cette cohérence atteignait plus de $60 \%$. 
de Cent Ans, un mythe créé peut-être dès le début de l'époque Moderne ${ }^{112}$ et qui fut par la suite véhiculé par de nombreux historiens.

Qu'en fut-il réellement, de cette nouvelle stabilité, voulue et affirmée? Pour répondre à cette question, nous devrons tenter d'appréhender la société vendômoise à travers d'autres sources, non écrites cette fois. L'étude des armoiries ${ }^{113}$ ou des demeures seigneuriales pourrait se révéler extrêmement riche : Bruno Phalip a montré l'importance de la signification sociale de l'architecture ${ }^{114}$. Les archéologues ont souvent tenté de retrouver sur le terrain les réalités décrites par le dénombrement. Or, la comparaison trop stricte de ces deux données ne s'est pas toujours révélée être opératoire mais il est probable qu'acte écrit et architecture du bâti tendaient à véhiculer un même message, une même vision du monde. C'est elle qui doit servir de fil directeur et permettre de lire les sources médiévales dans toute leur diversité.

112. La lecture " pessimiste " de Claude de Seyssel nous laisse entrevoir un groupe noble sur la défensive, menacé de décadence, qui vendait ses seigneuries à des officiers de finances ou de justice. ConTAminE, Philippe, "L'État et les aristocraties ", ConTAminE, Philippe (dir.), L'État et les aristocraties, XII - -XVII ${ }^{e}$ siècles, France, Angleterre, Écosse. Actes de la table ronde organisée par le Centre national de la Recherche scientifique, Maison française d'Oxford, 26 et 27 septembre 1986, Paris, Presses de l'École normale supérieure, p. 11-26.

113. Bony, Pierre, "L'image du pouvoir seigneurial dans les sceaux : codification des signes de la puissance de la fin du $\mathrm{XI}^{\mathrm{e}}$ au début du XIII ${ }^{\mathrm{e}}$ siècle dans les pays d'Oïl ", Seigneurs et seigneuries au Moyen Âge, Actes du $117^{\mathrm{e}}$ Congrès national des Sociétés savantes, Clermont-Ferrand 1992, CTHS, 1993, p. 367-402; MERINDOL, Christian de, " Le pouvoir seigneurial et son expression. Le témoignage de l'emblématique. Essai sur la Lorraine ", Seigneurs..., op. cit., p. 403-440. La constitution d'un véritable " armorial vendômois " devrait être entreprise dans les brefs délais, en reprenant la méthode établie par Robert Nussard (NussaRD, Robert, L'Héraldique médiévale en Touraine, Marches d'armes IV, Paris, Le Léopard d'or, 1989).

114. Phalip, Bruno, Seigneurs et bâtisseurs. Le château et l'habitat seigneurial en haute Auvergne et Brivadois entre le XI et le $X V^{e}$ siècles, Clermont-Ferrand, Presses universitaires Blaise Pascal, 1993. Également SALAMAGNE, Alain, " Le symbolisme monumental et décoratif. Expression de la puissance seigneuriale ", Seigneurs..., op. cit., p. 440-458. 
Annexe - Nombre d'aveux consultés par décennie (total : 445)

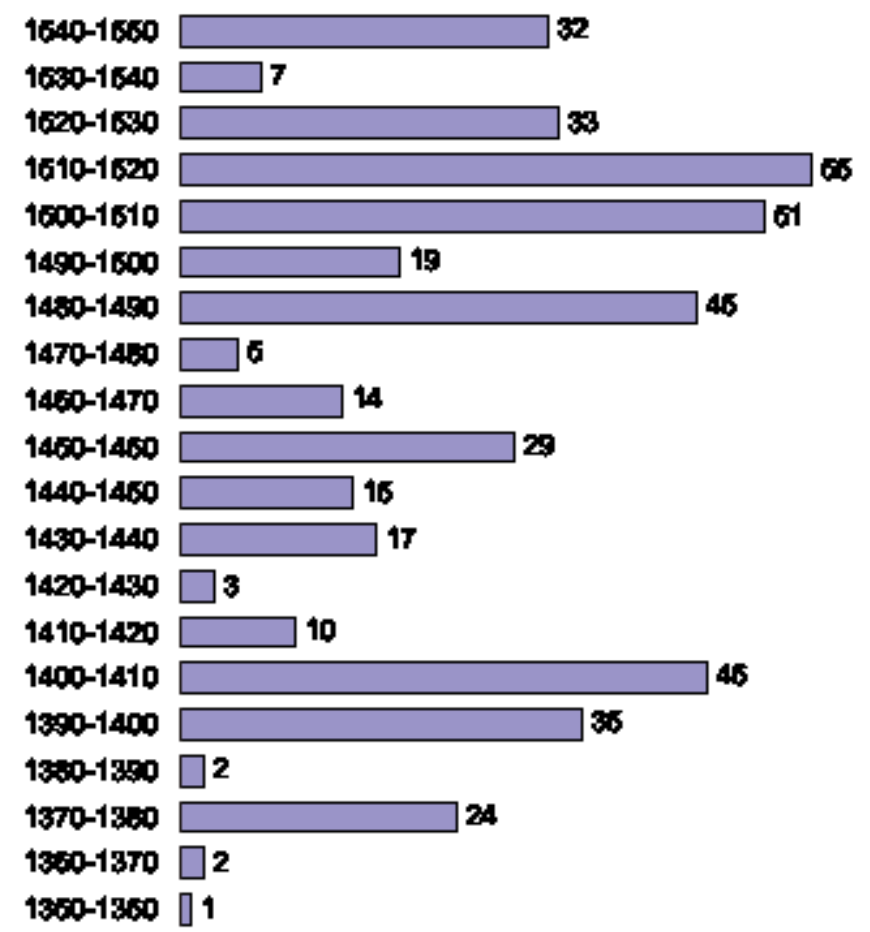




\section{RESUME}

Cette étude qui s'intéresse au Vendômois, du début du XIV ${ }^{\mathrm{e}}$ siècle au début du $\mathrm{XVI}^{\mathrm{e}}$ siècle, nous permet de comprendre comment le Comte de Vendôme s'est attaché à contrôler la haute société locale par l'aveu et dénombrement. Celui-ci, au début de notre période, nous fait découvrir un " état de noblesse " qui n'était pas affaire de titre mais de participation à un certain nombre de rites d'institution (au premier rang duquel figure le rite vassalique) qui permettait l'ascension sociale. L'élite, qui ne se dénommait pas noble, ne partageait pas les mêmes titres, était soudée par un même mode de vie. Le comte était quant à lui pressé d'octroyer une identité moins floue à chaque vassal : cette volonté mit quasiment un siècle et demi à triompher, aidée en cela par les progrès de l'écrit qui permettait de formaliser plus nettement la place de chacun. Ainsi, le statut de vassal devenait moins important que la référence à la " noblesse ", à la bourgeoisie ou à l'office judiciaire... L'écrit devenu véritable preuve n'affirmait plus seulement un droit sur la terre et les hommes mais également une noblesse davantage contrôlée par l'autorité comtale : ainsi triomphait sa vision d'un monde mieux défini, mieux surveillé et peutêtre plus fermé.

\section{ABSTRACT}

This study, which deals with the Vendômois, from the beginning of the XIV th century to the beginning of the XVI ${ }^{\text {th }}$ century, allows us to understand how the Count of Vendôme tried to control the local high society through the avowal and counting documents. The latter, at the beginning of the period we are concerned with, make us discover a "state of nobility" which was not a matter of title but of involvement in a certain amount of rites of institution (the vassalic rite ranking first) which made the social rise possible. The élite, which did not name, itself noble, did not share the same titles, was bound by a similar way of life. As for him, the count was keen to bestow a clearer identity on each vassal : it took this wish nearly one century and a half to prevail, with the help of the progress of writing which allowed to formalize more clearly the status of each one. Thus, the status of vassal became less important than the reference to "nobility", to bourgeoisie or to judicial office... Having become real evidence, writing not only asserted a right over land and men but also a nobility which was more controlled by the authority of the count : thus his vision of a world which was better defined, better supervised, and perhaps more exclusive prevailed. 